\title{
3D-Microfabrication of Polymer-Protein Hybrid Structures with a Q-Switched Microlaser
}

\author{
Sascha ENGELHARDT ${ }^{* 1}$, Yanlei HU ${ }^{* 2}$, Nadine SEILER ${ }^{* 2}$, Dominik RIESTER ${ }^{* 1}$, Wolfdietrich MEYER ${ }^{* 3}$, Hartmut \\ KRÜGER $^{* 3}$, Martin WEHNER ${ }^{* 2}$, Elke BREMUS-KÖBBERLING ${ }^{* 2}$ and Arnold GILLNER ${ }^{* 2}$ \\ ${ }^{*}$ Institute for Laser Technology, RWTH Aachen, Germany \\ ${ }^{* 2}$ Fraunhofer Institute for Laser Technology, Aachen, Germany \\ ${ }^{*}$ Fraunhofer Institute for Applied Polymer Research, Golm, Germany \\ E-mail: sascha.engelhardt@ilt.fraunhofer.de
}

\begin{abstract}
We demonstrate the feasibility of an inexpensive microchip laser source to fabricate 3D-polymer and protein microstructures based on two photon absorption of a commercial available photoinitiator. The voxel length was determined by an ascending voxel method and was as small as $1 / 4$ of the resolution limit. This technique was used to generate 3D polymer-protein-hybrid microstructures, where the polymer structure serves as a robust backbone for the protein structure.
\end{abstract}

DOI:10.2961/jlmn.2011.01.0012

Keywords: Multiphoton polymerization, scaffold, tissue engineering, extracellular matrix, serum albumin, photoinitiator, microchip laser, protein, polymer

\section{Introduction}

High resolution three-dimensional polymer structures are of great interest for various applications such as micro total analysis systems [1], microelectromechanical systems [2] and new photonic applications [3, 4]. These microstructures can be very useful and versatile in the field of tissue engineering. Cells, materials and engineering are combined in tissue engineering in order to improve or replace biological and biomechanical functions [5, 6]. The scaffold geometry and composition plays a major role in the effective generation of artificial tissue as it influences cell differentiation and proliferation [7]. Naturally derived scaffolds of extracellular matrix proteins best resemble the natural environment [8]. But the lack of mechanical stability often hinders the construction of more complex geometries, and thus non-biological polymers are used instead.

Complex three-dimensional structures can be constructed by means of multiphoton polymerization (MPP), which is a high resolution stereolithographic process [9]. The polymerization chain reaction of a prepolymer is initially triggered by a photoinitiator, which simultaneously absorbs two or more photons of a tightly focused and ultrashort pulsed laser beam. Polymerization only occurs at the focal point, where the laser intensity is strong enough.

The technique can be applied to biopolymers like extracellular matrix proteins, where cross linking between residue side chains is achieved via excitation of a photoinitiator [10]. A broad range of different proteins were successfully polymerized, including fibrinogen, concanavalin A and collagen [10-14]. Some proteins, such as serum albumin can be cross linked without the use of an additional (and often toxic) photoinitiator [13, 14]. In this case protein inherent aromatic amino acids (Phenylalanin, Tyrosin, Tryptophan) show a pronounced two photon absorption ability [15].

In order to use this process for generating complex three-dimensional microstructures a cost efficient and highly flexible MPP-machine was built. A comparable setup was used successfully for the microstructuring of bovine serum albumin (BSA) [13] and artificial polymers using specially designed photoinitiators $[16,17]$.

In this study a prepolymer solution based on an elastic polymer composed of a photo cross linkable oligomer and a reactive dilute was used with a commercially available photoinitiator to generate elastic 3D microstructures. The photoinitiator Irgacure ${ }^{\circledR}-184$ exhibits the maximum twophoton absorption cross section around $530 \mathrm{~nm} \mathrm{[18].}$ Microstructuring with $\mu$-chip laser systems and commercial photoinitiators has been proven feasible by analyzing the voxel length and generation of 3D polymer and proteinpolymer hybrid structures.

\section{Experimental Section}

\subsection{Material and Sample Preparation}

Bisphenol A ethoxylate diacrylate, lauryl acrylate (LA), isobornyl acrylate (IBA) were purchased from SigmaAldrich. The diacrylate resin $(50 \%)$ and the reactive dilutes (75\% IBA/LA) were mixed until 1\% Irgacure $^{\circledR}-184$ (kindly provided by Ciba AG, Switzerland) as photoinitiator was dissolved forming a photosensitive prepolymer (PP). The PP-composition was adapted so that the fully cured bulk 
material had a Young's modulus of $22 \mathrm{MPa}$ and elongation strength of $3 \mathrm{MPa}$.

BSA was purchased from Sigma-Aldrich. Prior to the experiments 200 or $400 \mathrm{mg}$ of BSA were diluted in $1 \mathrm{ml}$ distilled water without further use of photoinitiators.

The solutions used for fabrication were placed on a silanized glass slide, so that the polymer could adhere to the glass surface. 3-(Trimethoxysilyl)propyl methacrylate (Sigma-Aldrich) was used for silanisation according to the procedure provided by the supplier. A coverslip was placed over the solution in order to provide consistent sample conditions and to avoid evaporation. The sample height was also consistent by using $80 \mu \mathrm{m}$ thick spacers.

\subsection{Experimental Setup}

In order to generate three-dimensional cell scaffolds while using two-photon absorption (TPA), the following setup was used (Fig. 1). A small footprint, turn-key laser source (SNG-03E, Teem Photonics) with 550 ps pulse durations, $25 \mathrm{~mW}$ mean power and a repetition rate of $7 \mathrm{kHz}$ was used. The laser beam is attenuated by a diffractive attenuator, and expanded afterwards by a set of lenses. Approximately $8 \%$ of the beam intensity is then directed by a reflective pellicle onto a photodiode (S121C, Thorlabs) for online measurement of the applied laser intensity. The irradiation time is controlled by a mechanical shutter (BP108, Thorlabs). The beam is reflected by a high-reflective mirror into a microscope objective (20X, NA 0.5, Olympus), slightly overfilling the back aperture. Online monitoring and positioning is ensured by placing a charge-coupled device (uEyelE, IDS Imaging Development) behind the reflective mirror. A three axis linear stage system (ALS130, ATS100, Aerotech) is used for three dimensional sample positioning. The stage system, the CCD-camera, the shutter and the sensor are all controlled by a LabVIEW (National Instruments, Germany) program written in house.

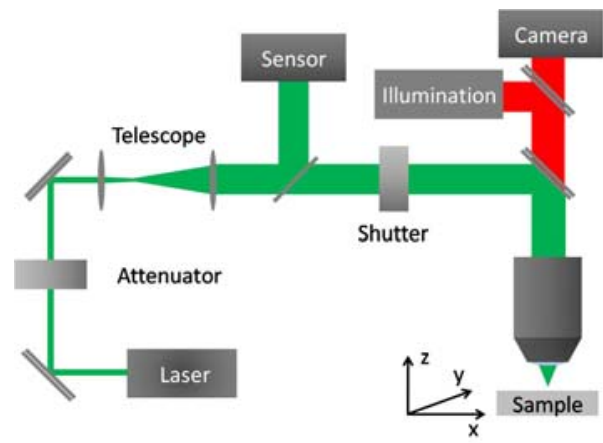

Fig. 1: The setup used for polymerization experiments, including a laser source, a telescope, a sensor for online power measurement, a mechanical shutter, a CCD camera and a microscope objective. The setup was controlled with a LabVIEW program.

\subsection{Determination of Voxel size}

The ascending voxel method was used to determine the voxel size [19]. A series of voxels was polymerized under the same $50 \mathrm{~ms}$ irradiation conditions but the height of the focal plane was increased for each consecutive voxel by $100 \mathrm{~nm}$. In order to circumvent additional shrinkage effects due to evaporation, especially for the protein voxels, the samples were analyzed prior to development with a light microscope using differential interference contrast microscopy. The voxel height was measured by counting the number of voxels present on the glass slide. SEM analysis further proves the method's validity. As individual voxel sizes can be smaller than the resolution limit of the microscope, images obtained with SEM confirm the number of voxels obtained by light microscopy.

\subsection{Matrix Fabrication}

Matrix fabrication was achieved by first creating a 3D model in computer aided design software (Rhinoceros 4.0, McNeel Europe) and subsequently translating it into machine code with computer aided manufacturing software (DCAM 5, S.K.M. Informatik).

For polymer matrices the non-crosslinked prepolymer is washed off using a solution of $50 \%$ ethanol/distilled water. BSA matrices were developed using distilled water. The solution was poured onto the irradiated sample, shaken gently for approximately 2 minutes and then dried at room temperature for one hour. These process steps were done subsequently for polymer-protein hybrid matrices. The polymer matrices were fabricated and developed first. The BSA solution was then added so that the BSA structure could be fabricated and developed. The samples were dried overnight at room temperature for further analysis.

\section{Results and Discussion}

MPP is based on a nonlinear optical effect of multiphoton absorption. Multiphoton absorption depends on the $\mathrm{n}^{\text {th }}$ power of the photon density. In the case of two photon absorption (TPA), the effective absorption focal area depends quadratically on the laser intensity, thus decreasing the effective polymerization volume. The propagation of the polymerization reaction upon TPA can be estimated by the amount of monomer that is converted to polymer by a radical polymerization chain reaction [17]:

$$
\frac{1}{\tau}=\frac{k_{p}}{\sqrt{2 k_{t}}} \sqrt{\frac{\Phi \sigma_{T P A}[A] F}{2(\hbar \omega)^{2}} \int_{p u l s e} I^{2} d t}
$$

Where $1 / \tau$ is the rate of polymerization, $k_{p}$ is the rate constant for polymerization propagation, $k_{t}$ is the rate constant for termination, $\Phi$ is the quantum yield for radical generation, $\sigma_{T P A}$ is the TPA cross section, [A] is the photoinitiator concentration, $\mathrm{F}$ is the repetition rate of the laser source and $\hbar \omega$ is the photon energy. Thus, the rate of polymerization can be expressed by $K_{i}$ which describes the material properties and $K_{l}$ which describes the laser source parameters and irradiation geometry [17]:

$$
\begin{aligned}
& K_{i}=k_{p}^{2} \Phi \sigma_{T P A}[A] / 2 k_{t} \\
& K_{l}=F / 2(\hbar \omega)^{2} \int_{\text {pulse }}^{I^{2} d t}
\end{aligned}
$$

The efficiency of different laser sources, that use the same optical setup, can be compared with the parameter $K_{l}$ from equation 2. A setup used for MPP by Serbin et al. [20] consisted of a femtosecond laser source with a wavelength of $780 \mathrm{~nm}$, pulse duration of $80 \mathrm{fs}$, repetition rate of 80 $\mathrm{MHz}$ and a mean power of approximately $30 \mathrm{~mW}$. The 
setup used in this study delivers laser pulses at a wavelength of $532 \mathrm{~nm}$, with pulse durations of $550 \mathrm{ps}$ and a repetition rate of $7 \mathrm{kHz}$. The maximum mean power measured after

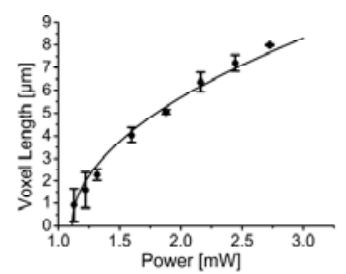

(a)

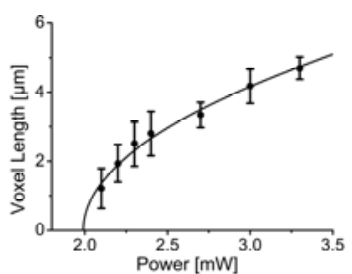

(b)
Fig. 2: The measured voxel length over the applied power for $50 \mathrm{~ms}$. The lines represent a fit of the experimental data for (a) the polymer and (b) the BSA solution with a model for TPP. The obtained voxel length can be tuned to values smaller than the resolution limit of the microscope objective $(4.3 \mu \mathrm{m})$ by an appropriate use of laser power and irradiation time.

the microscope objective was $13 \mathrm{~mW}$, although polymerization was observed also for much lower powers. A microscope objective with a numerical aperture (NA) of 0.5 results in $K_{l}=1 \times 10^{62}$ photons ${ }^{2} \mathrm{~m}^{-4} \mathrm{~s}^{-2}$ for the fs-setup and $K_{l}=8 \times 10^{61}$ photons ${ }^{2} \mathrm{~m}^{-4} \mathrm{~s}^{-2}$ for the ps-setup. Thus, the difference of the two systems is small, which indicates that both systems can be used for MPP.

The size of the TPA initiated polymerized voxels is an important feature used for generating microstructures as it determines the capability of fabricating structures with sub diffraction limited resolution. Furthermore, the dependency of the voxel size from the laser intensity indicates that the origin of the polymerization is TPA if a mathematical model derived for TPA can be applied. For radical polymerization the size of the voxels can be estimated by solving a rate equation by assuming that polymerization can only occur when a certain radical threshold is exceeded [20]. For TPA, the density of radicals depends on the photon flux squared $N_{0}^{2}$ and the irradiation time $t$, which leads to the following expression for the voxel length $l$ [20]:

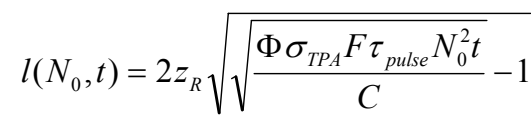

Where $z_{r}$ is the Rayleigh length of the laser beam and $C$ is a term describing the ratio of molecules accessible to radical formation and radicals necessary for initiating polymerization.

Assuming a Gaussian beam shape, equation 3 can be used to fit the experimental data and to obtain a value for $z_{r}$. As shown in figure 2 the theoretical predictions concur with the experimental data which indicates that the polymerization process is initiated by TPA. The minimum voxel length obtained was approximately $1 \mu \mathrm{m}$, which corresponds to a resolution of $1 / 4$ of the diffraction limit of the used microscope objective. The fitted values for $2 z_{r}$ are $5.8 \mu \mathrm{m}$ for the BSA solution and $6.3 \mu \mathrm{m}$ for the polymer solution. These values are close to the resolution limit of the microscope objective $z=2 \lambda n / N A^{2}=4.3 \mu \mathrm{m}$, for $n=1$. These results suggest that the observed process can be de- scribed by equations derived for two-photon polymerisation of a polymer system containing a commercial photoinitiator and a BSA solution without any additional

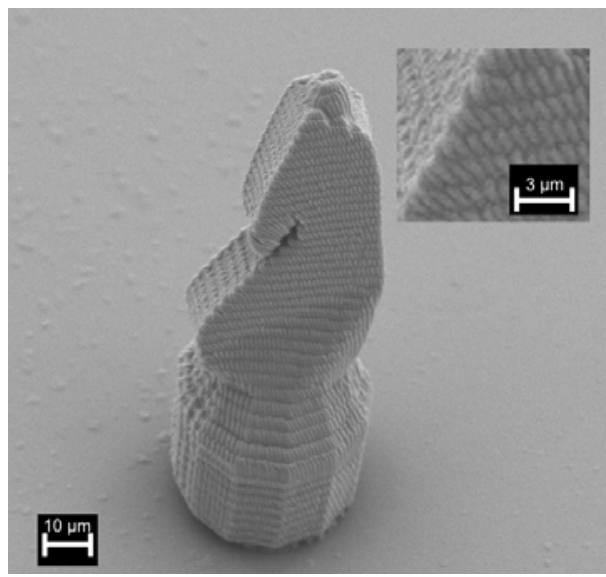

Fig. 3: SEM image of a micro-chess knight fabricated by two photon polymerization. A microchip laser source was used to polymerize an elastic polymer (PP) by radical polymerization initiated by a commercial photoinitiator.

\section{photoinitiator}

From figure 2 the threshold energy for TPA can be estimated. The threshold energies are $55 \mu \mathrm{J}$ for the polymer and $99 \mu \mathrm{J}$ for the BSA solution. Thus, the minimum irradiation time is approx. $7 \mathrm{~ms}$ for the polymer system and $15 \mathrm{~ms}$ for BSA.

For the generation of polymer-protein hybrid structure it is important that the damage threshold for each of the substances is higher than the corresponding threshold energies. Therefore the damage threshold of the polymer and the BSA solution was determined. For this reason the focus was positioned inside the solution to exclude an interaction with the glass surface and a $50 \mathrm{~ms}$ irradiation was applied with ${ }^{\mu \eta}$ arying power settings. Themdamage threshold was defined as the power setting where cavities started to form. This damage threshold for PP was $3.5 \mathrm{~mW}$ and for BSA $(400 \mathrm{mg} / \mathrm{mL}) 5.2 \mathrm{~mW}$, both values are close to the power required for polymerization. Thus, the parameters for matrix fabrication have to be chosen carefully. We chose a power level close to the polymerization threshold for the structures in this study. Although a BSA solution of 200 $\mathrm{mg} / \mathrm{ml}$ is suited for matrix fabrication, a protein concentration of $400 \mathrm{mg} / \mathrm{ml}$ was used. The higher concentration

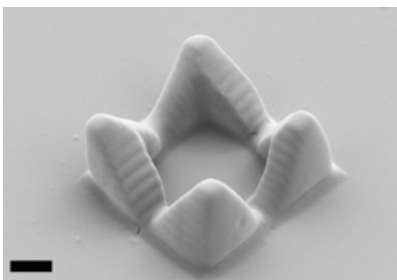

(a)

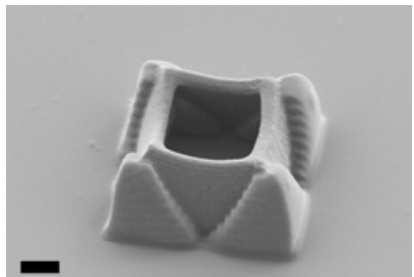

(b)
Fig. 4: (a) SEM image of a scaffold structure which serves as mechanical stable backbone for protein structures made from PP. (b) SEM image of a polymer-protein hybrid microstructure. The BSA rectangle retains it shape, although the sample has been thoroughly dried. 
leads to a higher process speed and a lower polymerization threshold. The power used for PP was $1.5 \mathrm{~mW}$, for BSA $2.1 \mathrm{~mW}$ and a writing speed of $50 \mu \mathrm{m} / \mathrm{s}$. The layer separation distance of the layer-by-layer approach was $2 \mu \mathrm{m} / 1 \mu \mathrm{m}$ and the distance between adjacent lines was $500 \mathrm{~nm} / 250 \mathrm{~nm}$ for PP/BSA. A micro-chess knight was generated as an example of 3D microstructuring while using a $\mu$-chip laser, PP and a commercial photoinitiator (see Fig. 3). The $2 \mu \mathrm{m}$ layer separation, as well as the $500 \mathrm{~nm}$ line separation distance and a substructure along the line propagation direction can be clearly seen. The substructure along the polymerized lines may be caused by the writing speed. Radical diffusion and accumulation can allow pearllike polymerization and wavy lines to develop, depending on the writing speed [21]. This effect would lead to the observed substructure.

An example of a polymer-protein hybrid structure can be seen in Figure 4. Here, a rectangular step structure of cured PP serves as a robust backbone for a BSA rectangle. The substructure described above is covered by a BSA residue from the development process. Nevertheless, the $2 \mu \mathrm{m}$ layer distance can be seen for the cured PP and the $1 \mu \mathrm{m}$ layer distance for the BSA structure. The BSA microstructure showed good adherence to the cured PP, since the BSA structure was still attached to the PP structure for every sample that was generated.

We demonstrated the technique for the generation of $3 \mathrm{D}$ polymer-protein hybrid structures with BSA, because it is inexpensive, easy to handle and curable without the need of an additional photoinitiator. Since the polymerization chemistry of proteins is similar, this method can be applied to other polymer/protein systems capable of MPP, which will be shown in future studies.

Such structures can combine the biological beneficial characteristics of extracellular matrix proteins with the stability of mechanically tuned artificial polymers. Furthermore, the prerequisite on the biofunctionality of artificial polymers would be lowered, since the protein structure could adopt most of these properties. This combination broadens the possibilities of tissue engineering in the generation of a functional cell scaffold.

In general MPP processes are slow compared to other rapid prototyping techniques for tissue engineering, but exhibit higher possible resolutions [22]. Thus MPP is useful in applications where such high resolutions may be beneficial. The process as it is presently described in this study is too slow to be used for macroscopic applications, such as a cell scaffold. However, there exist different possibilities for increasing the throughput. First, the use of highly efficient photoinitiators can be used to increase polymerization efficiency [23] and thus increase fabrication speed. Second, multi-focus techniques, like multi lens array [24] or phase modulation based processes [25] have been shown to improve process velocity. And third, a process parallelization by applying several setups capable of MPP can be used. For most setups capable of MPP only the first two possibilities are feasible, since the cost of a single MPP machine is too high. In this study we have demonstrated the potential of a cost efficient setup, based on a turn-key and small footprint $\mu$-chip laser. Thus, all three methods can be combined in the future to increase the fabrication throughput to a level where macroscopic scaffolds are possible in an adequate amount of time.

\section{Conclusion}

We have shown that MPP is possible using a small footprint, inexpensive $\mu$-chip laser system and a commercial available photoinitiator. This result demonstrates that the use of such a laser system does not necessarily require special synthesized photoinitiators, which makes it feasible for $3 \mathrm{D}$ microfabrication. We have further shown that artificial polymers and proteins can be used to generate threedimensional microscale hybrid structures. In the future, such structures can be used in complex cell scaffolds for cell guidance or for biological studies. We will use this technique to generate hybrid scaffolds with adapted properties.

\section{Acknowledgments}

We thank Aaron Rainer Elliott and Philipp Karbach for LabVIEW programming and Jan Timpe and Clemens Belle for the SEM images. The author gratefully thank the Fraunhofer Society for financing this research.

\section{References}

[1] P. S. Dittrich, K. Tachikawa, and A. Manz: Anal. Chem., 78, (2006) 3887.

[2] P. Grabiec, K. Domanski, P. Janus, M. Zaborowski and B. Jaroszewicz: Bioelectrochem., 66, 1-2, (2005) 23-28

[3] I. Staude, M. Thiel, S. Essig, C. Wolff, K. Busch, G. von Freymann and M. Wegener: Opt. Lett., 35, (2010) 1094-1096.

[4] J.K. Gansel, M. Thiel, M.S. Rill, M. Decker, K. Bade, V. Saile, G. von Freymann, S. Linden, and M. Wegener: Science 325, (2009)1513-1515.

[5] J. Lee, J. M.J. Cuddihy, and N.A. Kotov: Tissue Eng. Part B, 14, (2008) 61-86.

[6] D.W. Hutmacher, S. Cool: J. cell. and Mol. Med., 11, (2007) 654-669.

[7] J. Rouwkema, N.C. Rivron and C.A. van Blitterswijk: Trends Biotechnol., 26, (2008) 434-441.

[8] E. Filova, F. Straka, T. Mirejovsky, J. Masin and L. Bacakova: Physiol. Res., 58, (2009) 141-158

[9] K. S. Lee, R. H. Kim, D.Y. Yang, S. H. Park: Prog. Polym. Sci., 33, (2008) 631-681.

[10]J.D. Pitts, P.J. Campagnola, G.A. Epling and S.L. Goodman: Macromol., 33, (2000) 1514-1523

[11] S. Basu and P.J. Campagnola: J. Biol. Mat. Res., 71(A), (2004) 359-368.

[12] R. Allen, R. Nielson, D.D. Wise and J.B. Shear: Anal. Chem 77 (2005) 5089-5095.

[13]B. Kaehr, N. Ertas, R. Nielson, R. Allen, R.T. Hill, M. Plenert, and J.B. Shear: Anal. Chem., 78, 9, (2006) 3198-3202.

[14] M. Iosin, O. Stephan, S. Astilean, A. Dupperay and P.L. Baldeck: J. Optoelectron. Adv. Mater., 9, (2007) 716-720.

[15] A.A. Rehms and P.R. Callis: Chem. Phys. Lett., 208, (1993) 276-282. 
[16]C. Martineau, R. Anemian, C. Andraud, I. Wang, M. Bouriau and P.L.Baldeck: Chem. Phys. Lett., 362, (2002) 291-295.

[17]I. Wang, M. Bouriau, P.L.Baldeck, C. Martineau and C. Andraud: Opt. Lett., 27, (2002) 1348-1350

[18] K.J. Schafer, J.M. Hales, M. Balu, K.D. Belfield, E.W. Van Stryland and D.J. Hagan: J. Photochem. Photobiol., A, 162, (2004) 497-502

[19] H.B. Sun, T. Tanaka and S. Kawata: Appl. Phys. Lett., 80, (2002) 3673-3675

[20] J. Serbin, A. Egbert, A. Ostendorf, B.N. Chichkov, R. Houbertz, G. Domann, J. Schulz, C. Cronauer, L. Frohlich and M. Popall: Opt. Lett. 28, (2003) 301-303.
[21]F. Qi, Y. Li, H. Guo, H. Yang, and Q. Gong: Opt. Express, 12, (2004) 4725-4730

[22] S. M. Peltola, F. P. W. Melchels, D.W. Grijpma and M. Kellomäki: Ann. Med. 40 (2008) 268-280

[23] G. Kumi, C. O. Yanez, K. D. Belfield and J. T. Fourkas: Lab on a Chip 10, (2010) 1057-1060

[24] J. Kato, N. Takeyasu, Y. Adachi, H-B Sun, S. Kawata: Appl. Phys. Lett. 86 (2005) 044102

[25] K. Obata, J. Koch, U. Hinze, and B. N. Chichkov: Opt. Express 18 (2010) 17193-17200

(Received: June 07, 2010, Accepted: February 07, 2011) 